\title{
Eosinophilic Fasciitis Associated with Myositis
}

\author{
Yuko Adachi $^{\mathrm{a}}$ Yoko Mizutani $^{\mathrm{a}}$ En Shu ${ }^{\mathrm{a}}$ Hiroyuki Kanoh ${ }^{\mathrm{a}}$ \\ Tatsuhiko Miyazaki $^{\text {b }}$ Mariko Seishima $^{a}$ \\ ${ }^{a}$ Department of Dermatology, Gifu University Graduate School of Medicine, and \\ ${ }^{b}$ Division of Pathology, Gifu University Hospital, Gifu, Japan
}

\section{Key Words}

Eosinophilic fasciitis $\cdot$ Exercise $\cdot$ Magnetic resonance imaging $\cdot$ Myositis

\begin{abstract}
Eosinophilic fasciitis is clinically characterized by symmetrical scleroderma-like indurations of the skin with pain. The histological features are fascial inflammation with lymphocytes and eosinophils as well as thickened and fibrotic fascia. Lymphocytic infiltration and degeneration of the underlying muscle are rarely observed. We report a 69-year-old Japanese woman who presented with multiple areas of glossy induration and painful peau d'orange-like lesions on the chest and four extremities. T2-weighted magnetic resonance imaging showed significant hyperintense thickening of the fascia of the lower extremities. Histopathological examination of a biopsy specimen from the induration showed marked fibrinoid degeneration of the fascia and the neighboring muscle with mixed cellular infiltration of lymphocytes and eosinophils. The predominant CD8 + lymphocytic infiltrates were observed by immunohistological study. A diagnosis of eosinophilic fasciitis with myositis was made. Oral administration of prednisolone and discontinuation of exercise significantly improved the lesions and pain.
\end{abstract}

(c) 2015 S. Karger AG, Basel

\section{Introduction}

Eosinophilic fasciitis, first described by Shulman in 1975 [1], is a rare scleroderma-like disease characterized by symmetrical and painful swelling with progressive indurations and thickening of the skin [2]. Extracutaneous manifestations may rarely occur and include symmetrical polyarthritis, tenosynovitis, esophageal dysmotility, pulmonary fibrosis, pericarditis and hematologic diseases [3]. Eosinophilic fasciitis sometimes appears after intense physical

KARGER 125:s $\quad \begin{aligned} & \text { Mariko Seishima } \\ & \text { Department of Dermatology } \\ & \text { Gifu University Graduate School of Medicine } \\ & 1-1 \text { Yanagido, Gifu 501-1194 (Japan) } \\ & \text { E-Mail marikoseishima@yahoo.co.jp }\end{aligned}$


Adachi et al.: Eosinophilic Fasciitis Associated with Myositis

exertion. The characteristic histological features are fascial inflammation with lymphocytes and eosinophils as well as thickened and fibrotic fascia. The inflammation and fibrosis may extend to the lower dermis, but lymphocytic infiltration and degeneration of the underlying muscle are rarely observed. We report a patient working as a yoga instructor who developed eosinophilic fasciitis on the chest and extremities histologically associated with myositis.

\section{Case Report}

A 69-year-old Japanese woman, who had practiced yoga for 30 years and had worked as a yoga instructor for the past 2 years, noticed induration of the skin on her chest for 13 months prior to consultation. She also noted exertional pain in the indurated areas, and the number and size of the skin lesions gradually increased. She denied fever, arthralgia, cough and Raynaud's phenomenon. She had no history of L-tryptophan or toxic oil product ingestion or statin medication use. On initial consultation, multiple areas of glossy induration (fig. 1a) and painful peau d'orange-like lesions (fig. 1c) were observed on the chest and four extremities (fig. 1b). Raynaud's phenomenon, sclerodactyly, pitting ulcer on the fingers and nail fold bleeding were absent. Laboratory examination revealed eosinophilia $\left(0.71 \times 10^{9} / \mathrm{l}\right.$, normal $<0.45 \times 10^{9} / \mathrm{l}$ ), but serum creatine kinase, myoglobin, aldolase, IgG, IgA and IgM levels were within the normal limits and antinuclear antibodies were negative. T2-weighted magnetic resonance imaging (MRI) showed significant hyperintense thickening of the fascia of the lower extremities (fig. 1d). Histopathological examination of a biopsy specimen from an area of induration showed marked fibrinoid degeneration of the fascia (fig. 2a) and the neighboring muscle (fig. $2 \mathrm{~b}$ ) with mixed cellular infiltration of lymphocytes and eosinophils. Immunohistochemistry manifested predominant CD8+ lymphocytic infiltrates (fig. 2c) as well as a limited number of CD4+ lymphocytes (fig. 2d). Eventually a diagnosis of eosinophilic fasciitis with myositis was made. Oral administration of prednisolone $(0.5 \mathrm{mg} / \mathrm{kg} /$ day $)$ and discontinuation of yoga significantly improved the lesions and pain. The prednisolone was gradually reduced to $0.3 \mathrm{mg} / \mathrm{kg} /$ day.

\section{Discussion}

There are no universally accepted diagnostic criteria for eosinophilic fasciitis. According to the diagnostic criteria proposed by Pinal-Fernandez et al. [4], the patient fulfilled two major criteria, including clinical features and histological findings, and three minor criteria, including eosinophilia $>0.5 \times 10^{9} / \mathrm{l}$, Groove sign and/or peau d'orange findings, and a hyperintense fascia on T2-weighted MRI. In addition, systemic sclerosis was excluded on the basis of clinical features, laboratory data and histological findings. Thus, a diagnosis of eosinophilic fasciitis was made. However, hypergammaglobulinemia, muscle weakness and elevated aldolase levels were not observed.

Eosinophilic fasciitis is histologically characterized by a fibrous and inflammatory thickening of subcutaneous septa, fascia and the perimysial collagenous scaffold [5]. Macrophages and CD8+ T lymphocytes are reported to be the predominant cells in the inflammatory infiltrate. Some CD8+ lymphocytes contain granzyme B, suggesting a cytotoxic cellular immune response in eosinophilic fasciitis, which could be triggered by infectious or environmental agents. Although eosinophil infiltrates are present in $69-75 \%$ of patients with eosinophilic fasciitis [4], they may be absent at the chronic stage and are not essential for the diagnosis $[6,7]$. In our patient, CD8+ T lymphocytes and eosinophils were present in the infil- 
Adachi et al.: Eosinophilic Fasciitis Associated with Myositis

trate. Eosinophils infiltrating the fascia degranulate locally, resulting in release and accumulation of cationic granules with toxic and potentially fibrogenic properties in the tissues [4]. In the patient with both eosinophilic fasciitis and eosinophilic cellulitis, an abnormal circulating $\mathrm{T}$ cell clone and increased interleukin 5 production have been observed, suggesting that this phenomenon might be responsible for eosinophilia and eosinophil-mediated tissue injury [3].

Interstitial myositis has been observed in $68 \%$ of patients with eosinophilic fasciitis [8], but clinical myositis and muscle degeneration are rarely reported [2]. In the present case, muscle degeneration and inflammatory cell infiltrate involving the muscle were observed. In our case the degree of myositis was limited, and serum creatine kinase, aldolase and myoglobin levels were within normal ranges. In some cases of eosinophilic fasciitis, elevated creatine kinase levels [8] and abnormal electromyographic changes have been reported [5].

MRI of the involved muscle is considered useful for diagnosis of eosinophilic fasciitis [9]. Typically, in the acute phase of the disease, muscle MRI shows a markedly increased signal intensity within the fascia on fluid-sensitive sequences and a remarkable fascia enhancement after gadolinium administration in up to $80 \%$ of patients [10]. In the present case, MRI of the lower extremities showed a significant hyperintense, thickened fascia. Thus, MRI is recommended for the diagnosis of eosinophilic fasciitis.

Involvement of the trunk and morphea-like skin lesions are recognized risk factors for the development of persistent fibrosis and resistance to therapies [7]. In spite of a good response to systemic steroid therapy thus far, long-term physical therapy and follow-up are needed in the present patient, who had both truncal involvement and morphea-like skin lesions.

Eosinophilic fasciitis can be triggered by drugs, toxic exposure, physical exertion, bacterial infection and associated diseases such as autoimmune diseases, hematologic diseases and solid neoplasms. Among these, $30-46 \%$ of eosinophilic fasciitis patients have a history of intense physical exertion or trauma associated with the onset of the disease [2,6]. The present patient had practiced yoga for 30 years and had been a yoga instructor for 2 years before the onset of symptoms. In yoga, individuals maintain specific postures and perform exercises involving stretching of the extremities and bending of the trunk and/or extremities. The exercises are usually synchronized with breathing and are practiced by many for health and relaxation. There have been no previous reports of eosinophilic fasciitis induced by yoga. Although the affected area of the skin overlaid muscles being exercised in the present case, it is unclear whether the skin eruptions were caused by exercise.

\section{Disclosure Statement}

The authors declare that they have no conflict of interest and received no funding.

\section{References}

1 Shulman LE: Diffuse fasciitis with eosinophilia: a new syndrome? Trans Assoc Am Physicians 1975;88: 70-86.

2 Lebeaux D, Sène D: Eosinophilic fasciitis (Shulman disease). Best Pract Res Clin Rheumatol 2012;26: 449-458.

-3 French LE, Shapiro M, Junkins-Hopkins JM, Wolfe JT, Rook AH: Eosinophilic fasciitis and eosinophilic cellulitis in a patient with abnormal circulating clonal T cells: increased production of interleukin 5 and inhibition by interferon alfa. J Am Acad Dermatol 2003;49:1170-1174. 
Adachi et al.: Eosinophilic Fasciitis Associated with Myositis

4 Pinal-Fernandez I, Selva-O'Callaghan A, Grau JM: Diagnosis and classification of eosinophilic fasciitis. Autoimmun Rev 2014;13:379-382.

5 Toquet C, Hamidou MA, Renaudin K, Jarry A, Foulc P, Barbarot S, Laboisse C, Mussini JM: In situ immunophenotype of the inflammatory infiltrate in eosinophilic fasciitis. J Rheumatol 2003;30:1811-1815.

6 Bischoff L, Derk CT: Eosinophilic fasciitis: demographics, disease pattern and response to treatment: report of 12 cases and review of the literature. Int J Dermatol 2008;47:29-35.

7 Endo Y, Tamura A, Matsushima Y, Iwasaki T, Hasegawa M, Nagai Y, Ishikawa 0: Eosinophilic fasciitis: report of two cases and a systematic review of the literature dealing with clinical variables that predict outcome. Clin Rheumatol 2007;26:1445-1451.

8 Lebeaux D, Francès C, Barete S, Wechsler B, Dubourg O, Renoux J, Maisonobe T, Benveniste O, Gatfossé M, Bourgeois P, Amoura Z, Cacoub P, Piette JC, Sène D: Eosinophilic fasciitis (Shulman disease): new insights into the therapeutic management from a series of 34 patients. Rheumatology (Oxford) 2012;51:557-561.

$\$ 9$ Dybowski F, Neuen-Jacob E, Braun J: Eosinophilic fasciitis and myositis: use of imaging modalities for diagnosis and monitoring. Ann Rheum Dis 2008;67:572-574.

10 Desvignes-Engelbert A, Saulière N, Loeuille D, Blum A, Chary-Valckenaere I: From diagnosis to remission: place of MRI in eosinophilic fasciitis. Clin Rheumatol 2010;29:1461-1464.
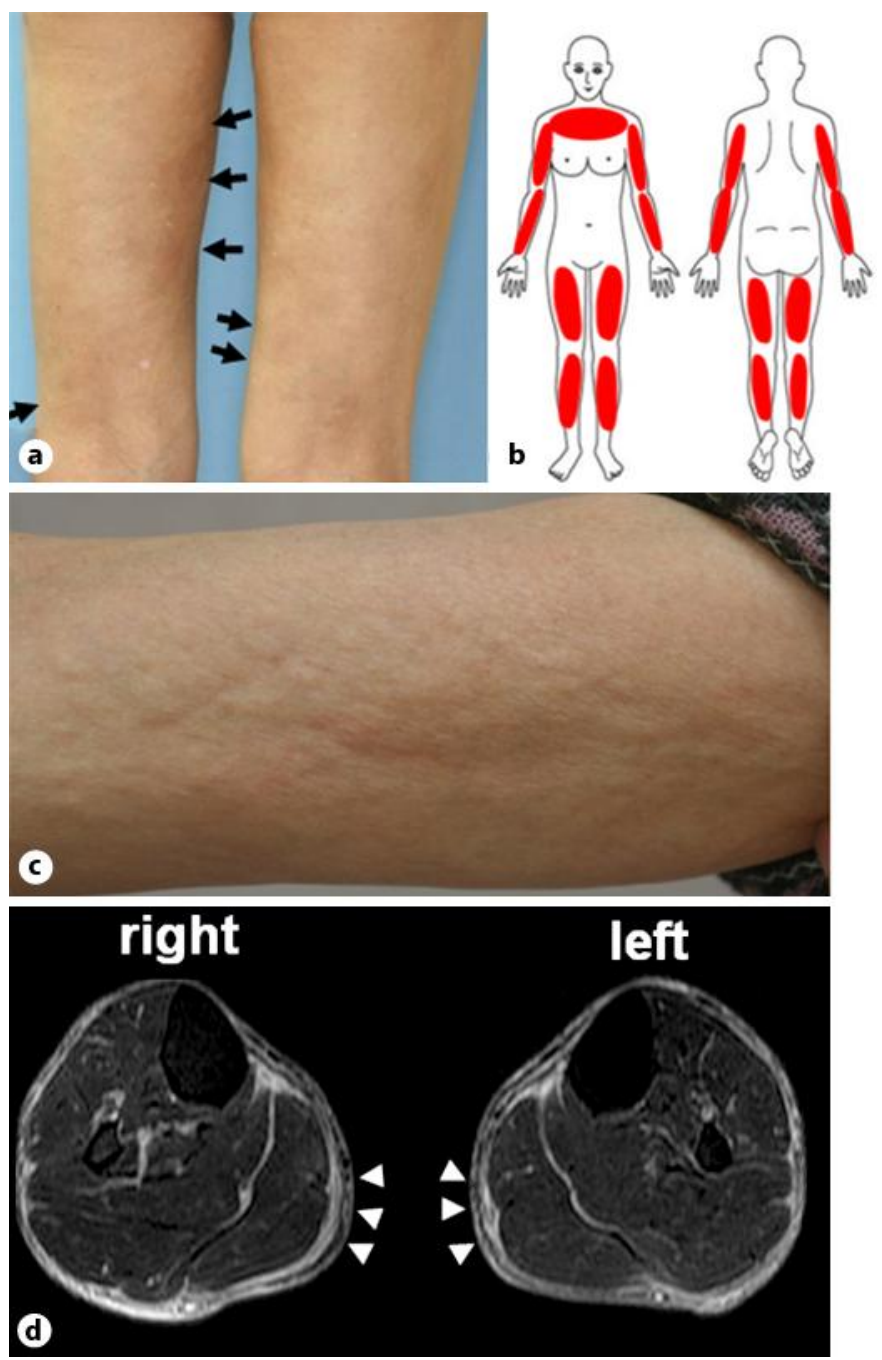

Fig. 1. a, c Areas of induration on the posterior thighs (arrows; a) and painful peau d'orange-like eruptions on the left upper arm (c). b Distribution of cutaneous induration (red color). d T2-weighted MRI showed significantly thickened fascia of the lower extremities (arrowheads). 


\section{Case Reports in Dermatology}

\begin{tabular}{l|l}
\hline Case Rep Dermatol 2015;7:79-83 \\
\hline DOI: 10.1159/000381845 & $\begin{array}{l}\text { C 2015 S. Karger AG, Basel } \\
\text { www.karger.com/cde }\end{array}$ \\
\hline
\end{tabular}

Adachi et al.: Eosinophilic Fasciitis Associated with Myositis

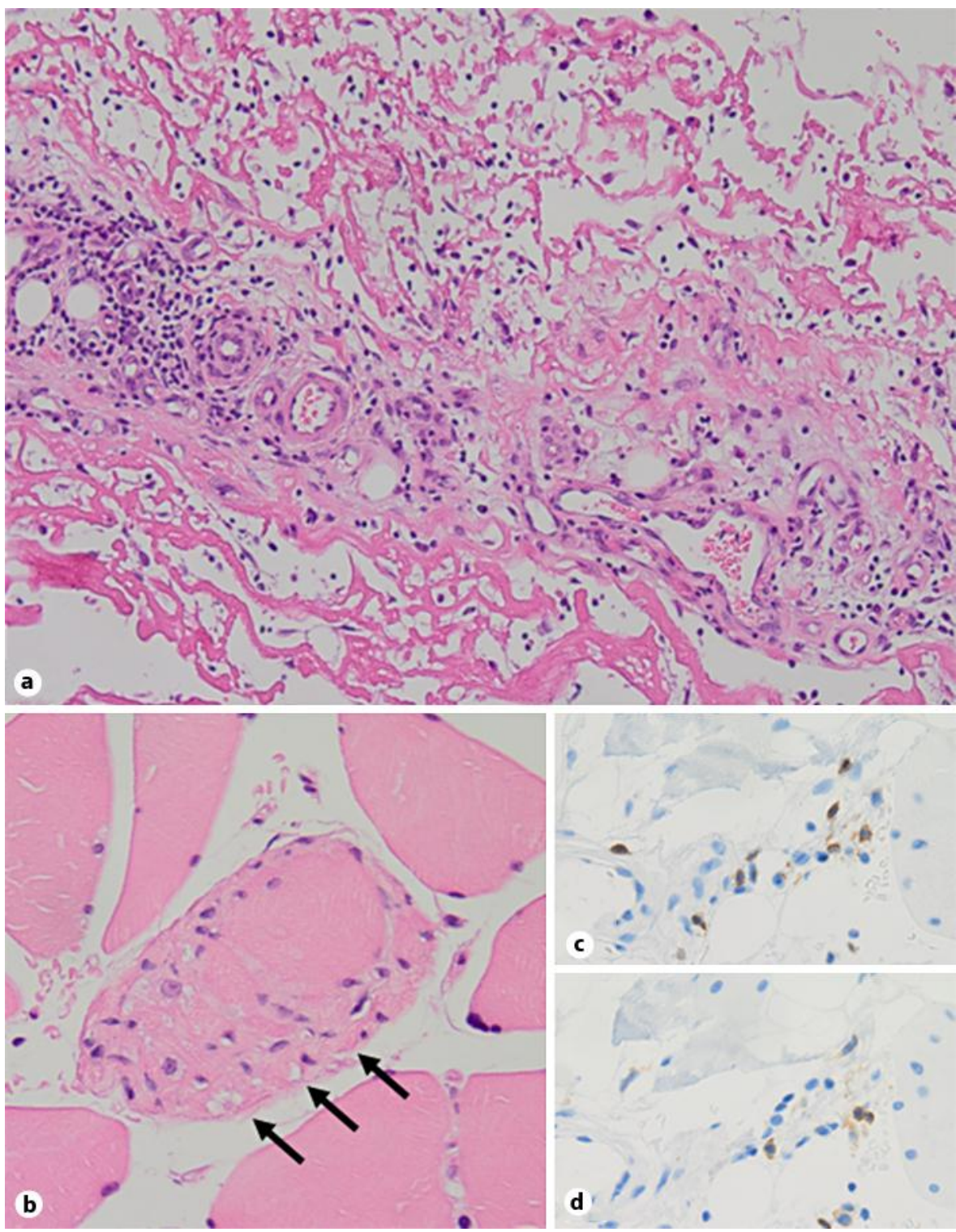

Fig. 2. a, b Photomicrographs of the induration (hematoxylin and eosin stain; original magnification: $\mathbf{a} \times 100, \mathbf{b} \times 200$ ). Degeneration of the fascia (a) and muscle (arrows, b) with infiltration of lymphocytes and eosinophils were observed. c, $\mathbf{d}$ Immunohistochemical stains by antibodies to CD8 (c) and CD4 (d) showing predominant CD8+ T cell infiltration (original magnification: $\times 400$ ). 